This is a peer-reviewed, accepted author manuscript of the following article: Urquhart, L. W. R., \& Wodehouse, A. (Accepted/In press). The emotive and semantic content of pattern: an introductory analysis. Design Journal.

\title{
The emotive and semantic content of pattern: an introductory analysis
}

This article examines the emotive significance of culturally prominent patterns and sets out an agenda for their purposeful use in design. An exploratory study reviewed 16 distinct patterns and asked participants to judge to what extent a set of emotion terminology related to the aesthetic of each. The results suggest the dynamics of observing aesthetically complex pattern are both exciting and interesting. Drawing on aesthetic theory, it is also argued that discrete geometric motifs within pattern may be subjectively related to emotions such as trust and fear. Connecting these motifs with specific concepts in aesthetic perception and interpretation, it is proposed that pattern can be selectively applied by designers hoping to develop emotively attuned product aesthetics.

Keywords: pattern design; emotion; form perception; semantics

\section{Introduction}

History of the aesthetic arts and ancient architecture reveals the prominence of decorative patterns of many different varieties. While there are clear historical reasons why patterns emerged as an artistic phenomenon, it is less apparent what the semantic properties of these forms were. What did they mean to the people of these times? This may be almost impossible to determine absolutely but considering the responses of modern-day people to culturally prominent patterns can inform our understanding, and provide useful insights for contemporary design practitioners hoping to attune products within specific emotional and semantic domains.

In the past, patterns developed along with other practices within the visual arts and now have a very rich history with much cross-cultural divergence. For example, the patterns of ancient Greece and Egypt were highly geometric and associated with forms of simple ornamentation (Pile, 2000), contrasting with later Celtic knot patterns which were much more explicitly organic, dominated by undulating curves used expressly for 
religious and devotional decoration (Frey \& Schwappach, 1973). Incredibly complex pattern design is also seen in the Islamic world incorporating a range of symbolism and ornate structural motifs which have subsequently been extremely aesthetically influential (Broug, 2013).

The nature of pattern and how we experience it opens several important questions relating to aesthetic experience more generally. Historically, aesthetic guides have emerged that seek to establish an objectivity to the visual experience. Vitruvius's De Architectura for example is one of the earliest to survive, dating to the $1^{\text {st }}$ century $\mathrm{BC}$ and describing the rules that characterise Classical architecture such as ornamentation, proportion and symmetry (Mitrovic, 1998). Later examples such as Hogarth's The Analysis of Beauty (1753) sought to deconstruct compositional elements to isolate what made them so visually appealing. This tradition of aesthetic analytics has continued into the present day along two distinct strands. One has examined aesthetics from a more philosophical and semantic perspective through art-historical narratives that consider social factors, aesthetic standards and phenomenology. The other strand is empirical and relates to the experience of the aesthetic on a cognitive, psychological or physiological level. The initial work questioning the nature of our perception postulated that our past experiences and situational context can greatly influence our reasoning capacities (Hume, 1738). Following the development of the psychological sciences, the questions raised by perception and visual experience have been continuously researched (Collier, 1996). Important work by early visual psychologists such as the Gestalt theorists established that compositional elements can redefine the meaning of a shape for an observer (optical illusion effects, for example) and more recent studies have begun to unpick the neurological explanations behind aesthetic preferences (Nanda, Pati, Ghamari, \& Bajema, 2013). 
Recent design research has worked to establish the emotive connection that develops when humans interact with objects and aesthetic compositions (see Jordan, 2002; Desmet, 2003; Hekkert, 2006; Hekkert \& Berghman, 2016). Pattern can be conceptualised in a similar way to designed objects, with both relying on established conventions of structural composition and can be a catalyst for emotional experience. Understanding its emotive relevance can have direct application in design, architecture and other visual languages such as film or graphic design. Increased interest in the emotive qualities of aesthetics within the context of design and styling sets a precedent for an exploration of this kind, where pattern can be applied directly to achieve a discrete experience. Additionally, Krippendorff and Butter (1984) and Krippendorff (2005) considered design elements from the perspective of semantic theory where meaning emerges within a nexus of signs and signals that can interact with an emotive interpretation, following the thinking of Saussure (1916/2013).

This research uses an exploratory study to address the emotive qualities of patterns, and uses an interpretivist approach to suggest possible semantic associations behind the emotive interpretations. Scholarly analysis of patterns has so far examined their underlying structure in terms of symmetry and geometry, and how this relates to kinds of cultural knowledge with notable contributions from Washburn and Crowe (1988) and Hann (2013). The study presented here builds on these past analyses and expands on recent work in experimental aesthetics which suggests that human beings have strong aesthetic preferences and associate particular emotive experiences with discrete geometry (Bertamini, Palumbo, Gheorghes, \& Galatsidas, 2016). Firstly, the aesthetic basis of pattern and the ways in which aesthetic form relates to emotional experience is examined. Secondly, the study explores a range of culturally prominent 
patterns and proposes that particular aesthetic motifs dependant on perceptual symmetry can be linked with distinct human emotions such as trust, fear and interest.

\section{What is a pattern?}

A pattern is a structured set of repeating aesthetic motifs. They have been seen in artistic practice for thousands of years with evidence of pattern decoration on ceramics dating back at least as far as the Neolithic period in Mesopotamia (Cruell, Mateiciucová, \& Nieuwenhuyse, 2017). Christie (1969, p.1) has noted that the term "pattern" implies design consisting of at least one element that is "multiplied and arranged in an orderly sequence". Considering that pattern creation relies on mathematical operations, the aesthetic changes in patterns have always been constrained by sets of rules, structurally bounded. Conventional tiling patterns consist of four fundamental elements: repeatability of forms, symmetry, complete coverage of a plane, and deconstructability. Indeed, repeating geometry that fits together, known in mathematics as a tessellation, is the central concept in pattern creation; what Christie (1969, p.77) has called processes of "interlacing, branching, interlocking and counterchanging". Hann (2012) details that patterns are built from four symmetry operations and these operations provide an emergent complexity when applied to arranged geometry (Figure 1).

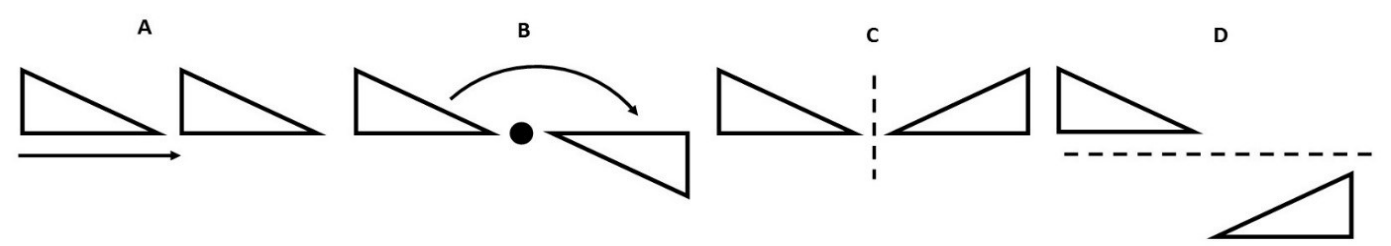

Figure 1: The four operations of symmetry; A) Translation B) Rotation C) Reflection D) Glide reflection 
Every pattern that perfectly covers a plane is built from these rules of symmetry that transform shapes. When a rule is applied to a shape or sets of shapes, a perfect tiling pattern can emerge. Depending on the arrangements of the shapes, great degrees of complexity can emerge. Seventeen two-dimensional configurations can exist to create a pattern in which a plane can be perfectly covered without varying any of the geometric elements. This relates to the maximal six-order rotational symmetry based on one of the five Bravias crystal lattice frameworks (Hann, 2012). While patterns can achieve high complexity, they can always be assessed within the bounds of symmetric rules. Interestingly, there is speculation that the use of complex symmetry operations in the context of pattern ornamentation is a kind of prehistoric group theory that became highly developed within ancient Egyptian and Babylonian civilization (Washburn \& Crowe, 1988). In this sense, pattern can be considered an expressive and aesthetic manifestation of mathematics.

The study and subsequent categorisation of pattern has been widely undertaken over the past two centuries. Owen Jones' study The Grammar of Ornament (1856/2008) is one of the first collections of the decorative aesthetics of world cultures. Jones not only details the contemporary design of his time but also historically significant pattern work from the Romans, Islamic and Byzantine peoples amongst others. He presents a series of seventeen "propositions" from which his theory of aesthetics if advanced. Proposition four for instance reads; "True beauty results from that repose which the mind feels when the eye, the intellect, and the affections, are satisfied from the absence of any want” (p.5). In its concluding chapter, Jones asserts that ornamental design should "go to nature as the Egyptians and Greeks went" (p.154) in an attempt to recapture beauty as he repudiates what he calls the "imitation" of nature that was present in the styles of his time. Interestingly, two of his closest disciples, the designer 
Christopher Dresser and architect Frank Lloyd Wright made pioneering use of organic ornamental styles in their respective ceramic and architectural works answering Jones' plea. Following Jones, Washburn and Crowe's extensive study Symmetries of Culture (1988) explored the mathematical basis of pattern design and also how this links to aspects of cultural knowledge - how symmetry relates to epistemic representations.

Analysis from anthropological research can show how important pattern work is to the practice of design. Ingold $(2008,2009)$ has extensively discussed processes of weaving, all of which are achieved by systematic patterning processes - the pattern creating both structural form and functionality through a process of gradual emergence. Knitting is another example of this. The making of rugs and carpets has a similar direct connection to patterning. Whether intentional or not, pattern is an emergent feature in design and construction. Brickwork is structured around a basic grid pattern with glide reflection symmetry, and car tyres are debossed by pattern-based texturing. Indeed, all manner of simple and complex patterning is present in everyday products. What, then, creates pattern? The most useful answer is shape. Ching and Juroszek (1998) define shape as an outline or configuration of surface form that can only exist with reference to other shapes. Structural elements are used to define and transform shapes within the context of a pattern such as the symmetry operations mentioned earlier. As Hann (2012; 2013) proposes, points (dimensionless entities) and lines are the basis of structure and are the sources from which all geometry flows. Line can be considered as a moving point or the path between two points, a change from one state to another or what Ingold $(2008,2015)$ has considered as flow of energy - patterns thus emerge from these flows interacting and interweaving. 


\section{Aesthetic perception, emotion and meaning}

Washburn and Crowe (1988) suggested that pattern design is an early experiment in group theory whereby aesthetic outputs are categorised into sets based on mathematical rules. In accordance with Cassirer's (1962) theory of aesthetic symbolism reflecting cultural beliefs, the creation of pattern in these ancient societies is evidence of a developing mathematical knowledge or a new epistemological framework. Some have suggested that the creation of patterns, and indeed other kinds of visual languages is a foundation for human reasoning capacities. Gestalt psychologist Rudolph Arnheim (1954) postulated that aspects of composition and geometric configurations within visual art can be used to understand aspects of human cognition where visual languages act as kinds of reasoning.

Recent work by Pinna (2010) has sought to extend the Gestalt principles to incorporate processes of meaning-making (semiotics). The Gestalt principles of grouping forms cannot fully explain the nature of these emergent meanings that manifest for observers, but there is what Pinna (2010, p.54) describes as a sense of "happening". This is an apparent change in the structure of a known form that is then ascribed a cause. Considering the forms in Figure 2, Pinna suggests that our natural response is to ascribe a cause for the deformation the square - it is being changed or influenced by some force. This is a method of cognitively processing the geometry. By giving the forms meaning and even some narrative of change, the geometry becomes an intelligible image. How an individual might process a pattern and ascribe meaning to it is affected by this and by what forms are considered visually dominant within a composition. Patterns are built from pieces of geometry and the context within which the geometry exists may affect interpretation. The interaction of shapes can create a visual energy, what Arnheim (1954) referred to as psychological forces. As such, a 
configuration of shapes may for instance appear "heavy" or "light" depending on its context.
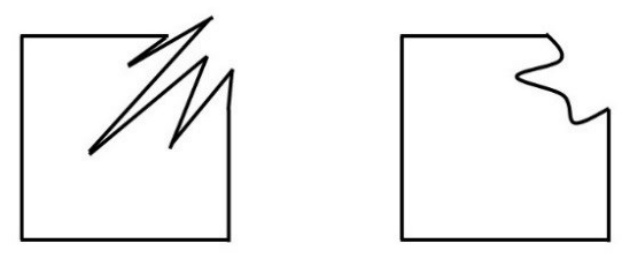

Figure 2: The proposed "sense of happening" affect, adapted from Pinna (2010)

The proposition that pattern serves both as a form of meaningful symbolism and has intrinsic use value has been explored by anthropologists. Work by Gell (1998) for example argues that the use of pattern in ancient cultures was an act of warning off the demonic. Evil spirits would be both tantalised and puzzled by patterns and would not enter a space if it could not be unravelled. Gell (1998) notes that Celtic knot work may have been used in this way and Ingold (2008) cites the cultures of southern India who also engaged in similar practices. This form of pattern making, Ingold argues, is analogous to, or representative of, a maze or a labyrinth and believes that instead of confusing demonic forces, pattern making is a practice of trapping them in an environment of repeating loops. These respective analyses are interesting as they suggest that pattern has both a semantic dimension, relating to Krippendorff (2006), but also a functional dimension following Gibson's affordance theory (1991). Ingold strongly argues that forms are not just observed in a prescriptive and rational way, they are modes of expressing change, flows of energy and material interactions. In this sense, pattern making cannot be understood as a static ornamental practice, the way Jones (1856/2008) viewed it, but a complex expression of cultural beliefs and historical journeys. The authors (2018) have previously proposed that elements of architecture and design can be viewed as arranged aesthetic archetypes that reflect dominant cultural 
ideas of certain historical periods. Here, aesthetic archetypes play a similar role that Jung (1991) theorised character or narrative archetypes have; relating to a symbolic higher (but ineffable) meaning.

Researchers within experimental aesthetics have also explored the experience of abstract geometry and attempted to establish what emotional links humans have with it in the visual domain. Studies have consistently shown that broadly curved stimuli are preferred over angular stimuli, and that discrete geometric elements influence overall interpretation. An early study by psychologists Poffenberger and Barrows (1924) showed that angular visual stimuli were associated with words such as "powerful", "serious" or "hard". Their experimental work used 18 variations of simple line drawings with wave-like forms, varying in frequency. Participants were asked to assign sets of adjectives to each. The curved visual stimuli were associated with words such as "gentle" and "quiet" meaning there was an important experiential distinction as people looked at the different lines. Similar results have been recorded more recently by Collier (1996) in a set of experiments, by Bar and Neta (2006), Silvia \& Barona, (2009) and also by Bertamini et al (2016). Recent developments in shape perception theory has focused on how distinct features are detected. Foster (1984), for instance, established that four features can be coherently studied with clear linked to the experience of shape recognition:

1) Recognition of local features - straight lines versus curved lines, acute versus obtuse angle differentiation

2) Local spatial recognition - shape arrangement within a reference plane

3) Global feature recognition - awareness of symmetry and orientation

4) Global spatial relationship recognition - awareness of the position of geometry within a given field

These criteria can help in the decoding of pattern perception given the importance of symmetry, orientation and geometric positioning has within pattern 
design. In a wide variety of contexts and experimental conditions, there is a broad association between form and emotional states. Considering the qualities of pattern aesthetics, we make the philosophical assumption that form can be meaningfully interpreted by observers but that this experience is guided by emotive drivers and culturally derived semantic interpretations. The aesthetic experience models developed by Leder and others $(2004,2014)$ instantiate this view.

\section{Human emotion and experience}

On what basis do we discuss emotion and the notion of experience? The work of pragmatist philosopher John Dewey (1934) considered the nature of aesthetic experience and is useful for our purposes. He postulated that the act of viewing a work of art goes beyond simply the formal properties of the work, operating with the emotions and sentiments of the viewer to create a discrete experience. An experience is an event that is demarcated by a beginning and an end and has a distinct and individual quality. How do we therefore consider emotions in the context of an aesthetic experience? Many theories on emotional experiences have been proposed in recent history (see Izard, 2009 for a detailed summary). Early thinking proposed that emotion was a function of an antecedent arousal state (James, 1884). Emotions have been described by Arnold (1960) as an adaptive function where emotions help the human animal to understand events through cognitive appraisal (Lazarus, 1991). Appraisal theory considers human emotion as a form of stimuli response where emotions developed within humans as a means of survival (Scherer, Schorr, \& Johnstone, 2001).

Work to classify emotions and the relationship between distinct emotive experiences was undertaken by numerous researchers (see Plutchik, 1980; Ekman, 1982; Shaver et al., 1987; Russell, 2003). Plutchik's (1980) model sought to show how emotive experiences can be highly transient and dependent upon other emotive states. 
For example, the intensity and character of feeling in the emotions we call "joy" is similar to that of "anger". What makes the experiences distinct is the contextual origin.. Similar models such as Russell (2003) have classified emotions in other distinct ways but still note distinct changeability and transience. Emotive classification must be applied to our examination of pattern as a highly coded means of articulating subjective experiences. The next sections articulate our exploratory study and our approach to examining emotive experiences of pattern aesthetics.

\section{Exploratory study}

16 patterns were selected from Wade's (1982) catalogue which identifies culturally significant pattern designs from across the world. This source was selected based on its clarity, excellent range of examples, and its structural breakdown of each pattern's design. During our research, the author David Wade, was contacted and granted permission for their use in the study. Given that it was unfeasible to analyse every possible type of pattern, the selection was limited to those showing only simple motifs built from concrete geometric shapes, avoiding complex symbolism or imagery. The patterns were also selected with the aim of presenting a wide range of geometry and structural arrangements to participants. While direct symbolism does constitute much pattern design, the Gestalt visual perception theories and the subjective construction of meaning was the main focus of exploration.

The patterns used for the analysis are shown in Figure 3. To assist post processing of the data, the patterns were categorised following their geometric makeup using a curvature versus angular dichotomy. 

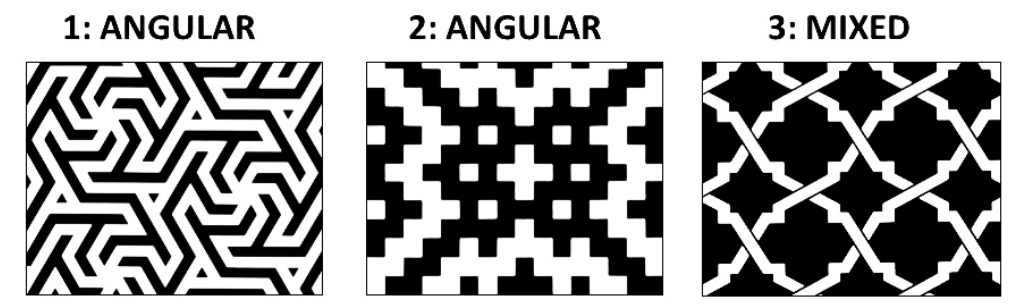

4: MIXED

5: ANGULAR

6: CURVED

7: MIXED
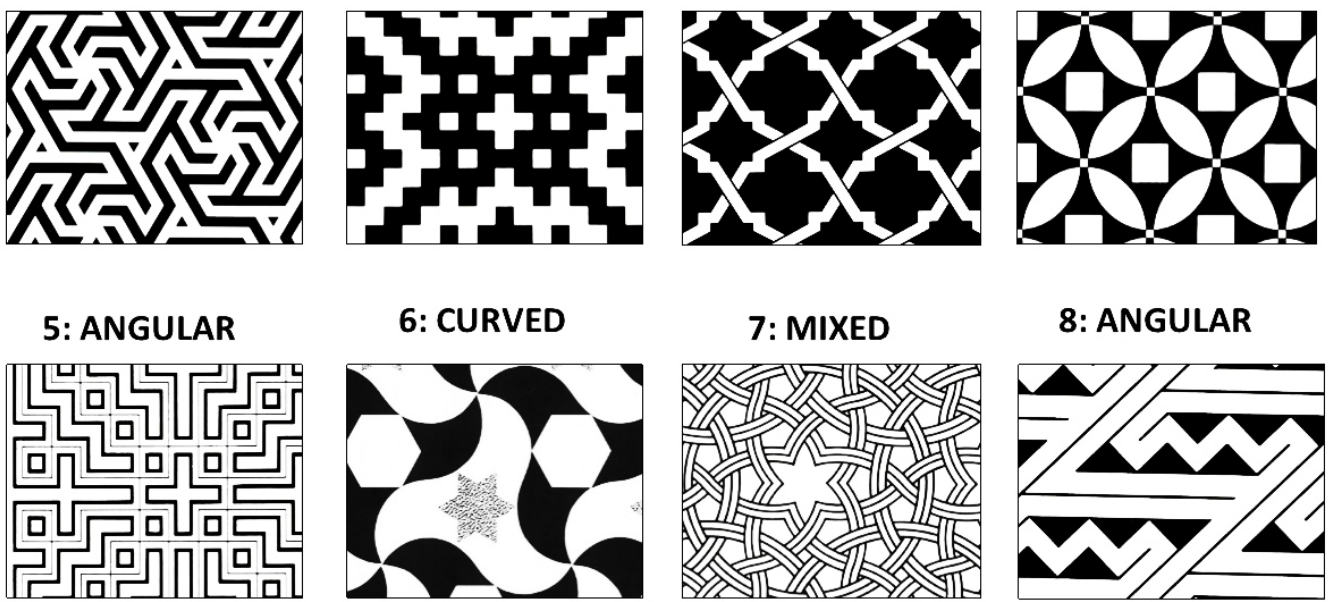

8: ANGULAR

9: ANGULAR

10: ANGULAR

11: CURVED

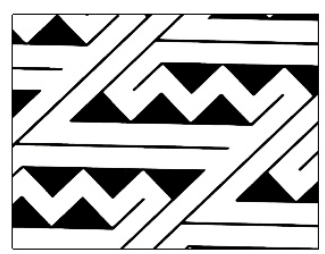

12: CURVED
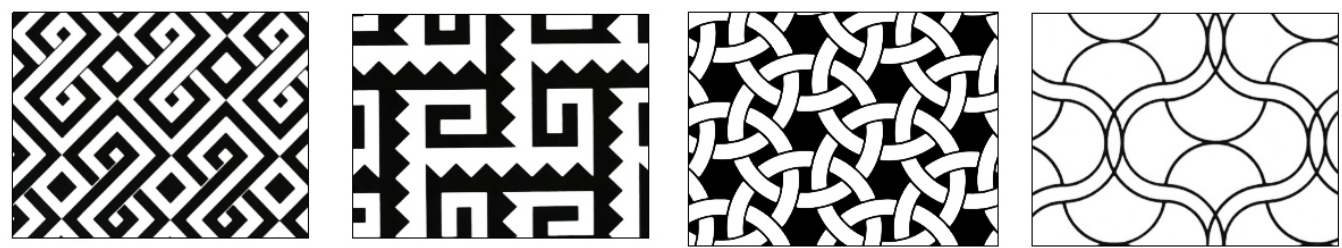

13: CURVED

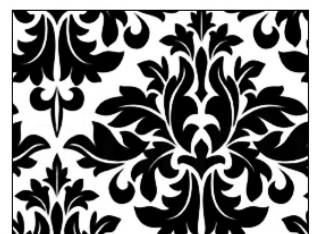

14: MIXED

15: CURVED

16: CURVED
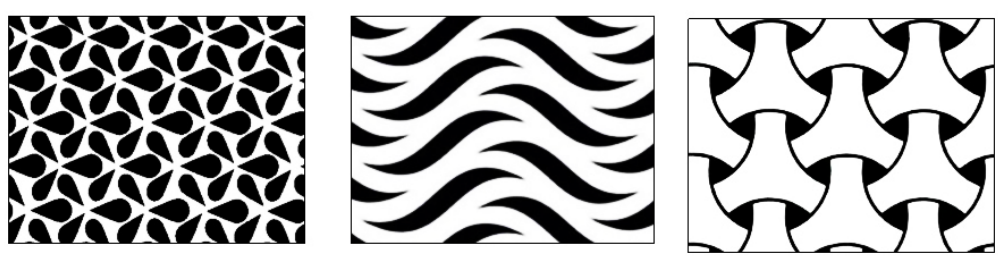

Figure 3: 16 patterns used for the study (Wade, 1982), reproduced with permission from author

\section{Methodology}

The study took an exploratory approach and incorporated a quantitative assessment of data in terms of participant responses. As described, the study considered 16 patterns from a variety of cultural sources and analysed them with respect to the subjective representation of emotion. A list of 16 emotive terms developed by Plutchik (1980) as semantic descriptors of primary human emotions was used alongside an ordinal scale of 0 to $5 ; 5$ representing an intense emotive representation and 0 indicating no emotive representation. The list of emotive terminology used for the experiment is presented in 
the left column of Table 1. 30 participants (16 females and 14 males) took the test individually. Every pattern was shown in turn for a maximum of 90 seconds in the same order as presented in Figure 3. For each pattern example, the participant indicated to what extent the pattern subjectively represented the emotion. Each pattern design was displayed on a 15-inch laptop screen with high-definition resolution. The overarching goal was to determine if particular varieties of pattern - those dominated by angular or curved forms or patterns with strongly identifiable visual motifs - could be linked to representations of emotion.

\section{Results of exploratory study}

Many of the patterns were interpreted in ways that conform to the existing paradigms in vision research (Table 1). There were however some interesting results that can be interpreted through other more theoretical means such as Gestalt psychology and other concepts in the study of design and emotion.

Table 1: Collective averages of emotive responses to 16 patterns

\begin{tabular}{|c|c|c|c|c|c|c|c|c|c|c|c|c|c|c|c|c|}
\hline Pattern & 1 & 2 & 3 & 4 & 5 & 6 & 7 & 8 & 9 & 10 & 11 & 12 & 13 & 14 & 15 & 16 \\
\hline Acceptance & 1.63 & 1.33 & 2.67 & 2.87 & 1.43 & 2.57 & 2.53 & 1.23 & 2.17 & 1.17 & 2.77 & 2.53 & 2.47 & 1.60 & 2.17 & 2.23 \\
\hline Anger & 1.60 & 1.57 & 0.87 & 0.47 & 1.70 & 0.40 & 0.93 & 1.53 & 1.17 & 1.57 & 1.00 & 0.33 & 0.63 & 1.03 & 0.83 & 0.83 \\
\hline Annoyance & 2.07 & 2.67 & 1.23 & 0.83 & 2.17 & 1.13 & 3.30 & 2.40 & 1.87 & 2.33 & 1.07 & 0.77 & 1.07 & 1.93 & 1.60 & 1.33 \\
\hline Anticipation & 1.93 & 1.83 & 1.57 & 1.40 & 2.07 & 1.70 & 2.37 & 1.83 & 2.10 & 1.87 & 1.77 & 1.70 & 1.60 & 1.53 & 1.57 & 1.37 \\
\hline Apprehension & 2.27 & 1.93 & 1.47 & 1.23 & 2.17 & 0.90 & 0.80 & 2.13 & 1.77 & 1.97 & 1.57 & 0.97 & 1.13 & 1.27 & 1.67 & 1.40 \\
\hline Boredom & 0.77 & 1.40 & 1.13 & 0.87 & 0.93 & 0.77 & 1.63 & 1.00 & 0.93 & 1.27 & 0.90 & 1.17 & 1.33 & 1.47 & 0.93 & 1.30 \\
\hline Disgust & 1.07 & 1.67 & 1.00 & 0.30 & 1.63 & 0.67 & 2.33 & 1.67 & 1.23 & 1.40 & 0.73 & 0.63 & 0.83 & 1.80 & 0.87 & 0.90 \\
\hline Distraction & 3.30 & 2.60 & 1.67 & 2.23 & 2.77 & 1.93 & 0.93 & 2.57 & 2.90 & 2.67 & 2.27 & 1.87 & 1.87 & 2.40 & 2.13 & 1.53 \\
\hline Fear & 1.27 & 1.37 & 1.20 & 0.40 & 1.47 & 0.57 & 0.57 & 1.97 & 1.13 & 2.07 & 0.90 & 0.60 & 0.77 & 1.17 & 1.13 & 1.20 \\
\hline Interest & 3.00 & 2.00 & 3.23 & 2.87 & 3.40 & 2.97 & 1.53 & 2.37 & 3.23 & 2.33 & 2.47 & 2.53 & 2.67 & 2.30 & 3.00 & 2.47 \\
\hline Joy & 1.43 & 1.00 & 1.87 & 2.23 & 1.20 & 3.03 & 2.33 & 1.00 & 1.73 & 0.77 & 2.10 & 2.40 & 2.30 & 1.53 & 1.67 & 1.63 \\
\hline
\end{tabular}




\begin{tabular}{|c|c|c|c|c|c|c|c|c|c|c|c|c|c|c|c|c|}
\hline Pensiveness & 2.47 & 1.93 & 2.47 & 2.77 & 2.73 & 2.10 & 2.67 & 1.77 & 2.07 & 1.83 & 2.17 & 2.20 & 2.03 & 1.53 & 1.77 & 2.13 \\
\hline Sadness & 0.87 & 1.27 & 1.63 & 0.83 & 1.37 & 0.90 & 1.83 & 1.37 & 0.90 & 1.20 & 1.20 & 1.17 & 1.00 & 1.20 & 1.07 & 1.13 \\
\hline Serenity & 1.17 & 1.10 & 2.17 & 2.67 & 0.87 & 2.83 & 2.20 & 0.93 & 1.53 & 0.80 & 2.10 & 2.40 & 2.17 & 1.40 & 2.50 & 1.40 \\
\hline Surprise & 1.73 & 1.50 & 1.73 & 1.70 & 2.37 & 1.90 & 0.83 & 2.53 & 2.50 & 2.33 & 1.90 & 2.70 & 2.23 & 1.47 & 1.80 & 1.27 \\
\hline Trust & 1.27 & 1.17 & 2.03 & 2.30 & 1.20 & 2.60 & 1.10 & 0.90 & 1.60 & 0.97 & 2.57 & 2.03 & 2.87 & 1.47 & 1.97 & 2.43 \\
\hline
\end{tabular}

\section{Discussion}

This section will discuss several key results that can give us some insight into the complexities of aesthetic perception with respect to pattern design. It should be noted that the work remains exploratory in nature, and as such is reliant upon the subjective interpretation of data. It is also limited in scale, totalling 30 participants, meaning that the conclusions offered are more speculative and theoretical. We employ interpretivist methods, following Panofsky (1938), viewing aesthetics not just as formal properties (proportion, composition etc.) but as subjective and symbolic representations where a perceptual semantic meaning is embedded.

\section{Patterns with curves are associated with positive emotions}

Overall, the results are consistent with the literature, finding that patterns containing a lot of curved geometry were preferred, and associated with characteristically positive emotions. More systematic research would however be needed to establish with more certainty some of the conclusions that are speculated within this section. Patterns 3, 4, $6,11,12$, and 13 are unambiguously positive for observers due to the high average rankings for the semantically positive emotions of acceptance, trust, interest, joy, and serenity seen across all the results. By contrast, low averages were found for the semantically negative emotions such as fear, as illustrated in Figure 4. Pattern 7, while being mixed in its form elements, showed high levels of annoyance (averaging 3.30) indicating its high score for joy cannot be considered as a concrete indicator of a 
positive interpretation. Similarly, for patterns 14,15 and 16 -while containing dominantly curved elements, the scores were unexpectedly low for joy, an indicator of a positive emotive interpretation, following the categorisations developed by Plutchik (1980) and Ekman (1982). Other semantically positive factors remained high, serenity and interest being notable examples, but these results suggest an ambiguity - some compositional element that hinders the positive interpretation. An answer might be that the angular elements are being viewed as more visually dominant in the compositions overall.

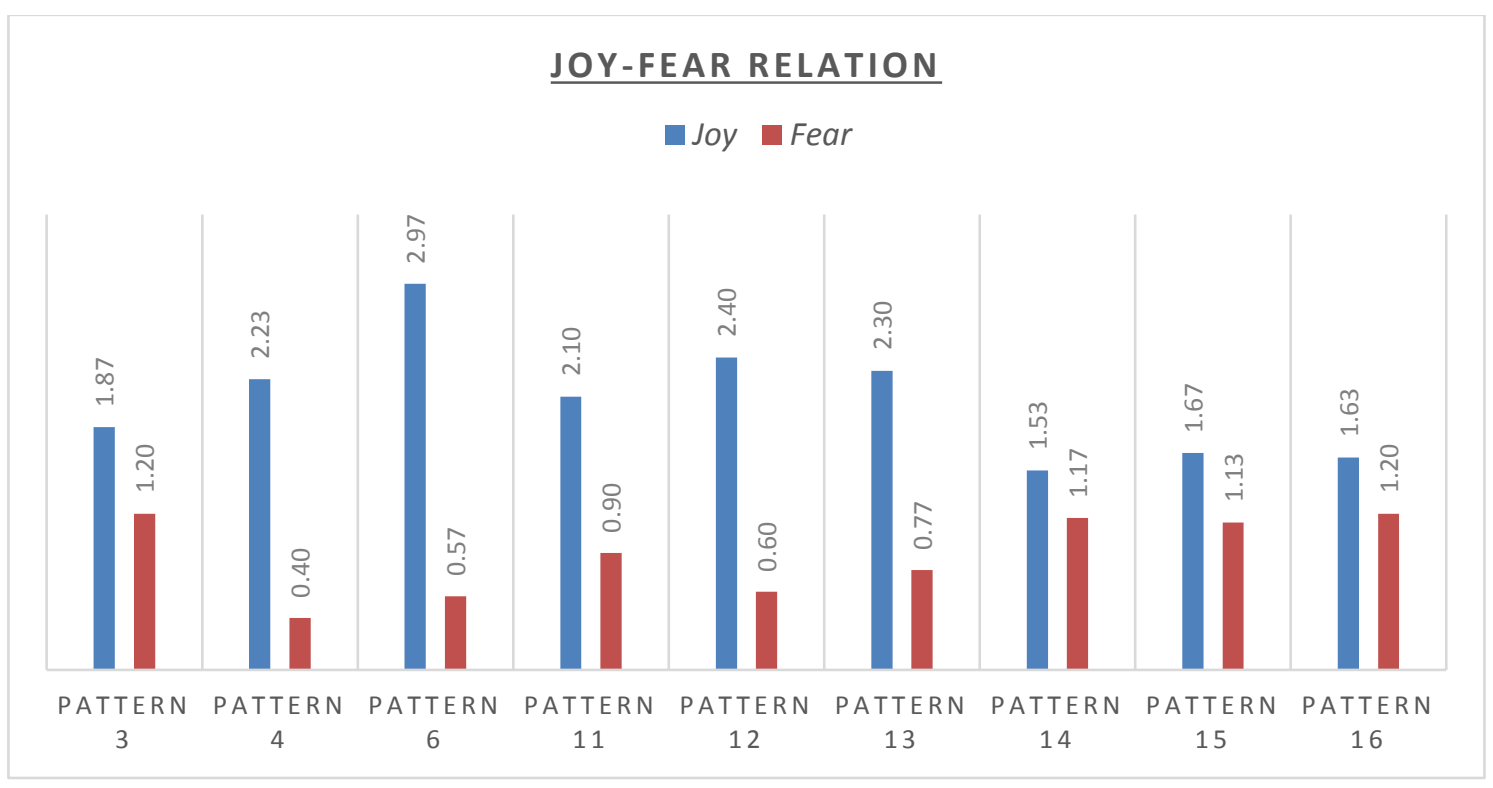

Figure 4: Joy-Fear comparison for curvature dominant and mixed geometry patterns

\section{Symbols of trust}

The compositional element of curved interlocking features appears to correlate to high scores for the emotion trust. It has previously been demonstrated that simple arrangements of lines can convey complex emotive meaning (Salgado-Montejo et al., 2017). As an aesthetic element, the interlocking rings may subjectively suggest a sense of rigidity and by extension reliability - a kind a symbolic functionalism. The rigid structure of a tied knot for instance or a set of chain-links all have thematic and 
aesthetic similarities. Perceptually, the strong symmetrical basis of this motif may also play a role where an obvious and identifiable symmetry leads to subjective ties to concepts of balance. An asymmetrical arrangement of rings would possibly have a different response. The symmetrical nature of the motif also allows for its use within pattern design - a perfect pattern utilising an asymmetrical variant would not be possible. Additionally, it is worth noting the unity of two rings (Figure 5) was used frequently in Christian High-Gothic architecture named the "Vesica Piscis" (Hiscock, 2007). It is possible that this cultural motif is influencing the emotive interpretation- of the patterns assessed to be high in trust, four contain this symbol in some manifestation (pattern 4, 11, 12 and 16). Following the thinking of Arnheim (1954), this symbol may reflect a deeply embedded trust-response within human experience and human reasoning.

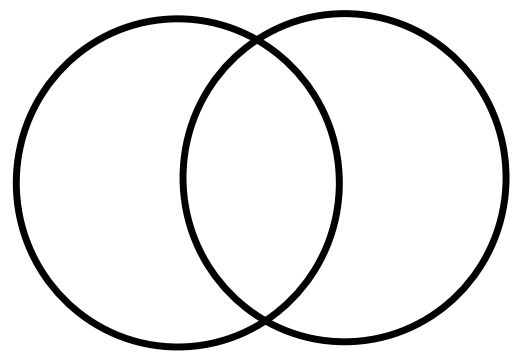

Figure 5: "Vesica Piscis" motif of overlapping rings is consistently associated with feelings of trust

There is also an apparent link between acceptance and trust responses (Figure 6). When a high score is recorded for trust, a high score for acceptance is also recorded, indicating a conceptual link that is perhaps made by the observers. The conceptual definition of trust suggests an implicit acceptance; trust in somebody or something means to accept or put faith in an aspect of it. 


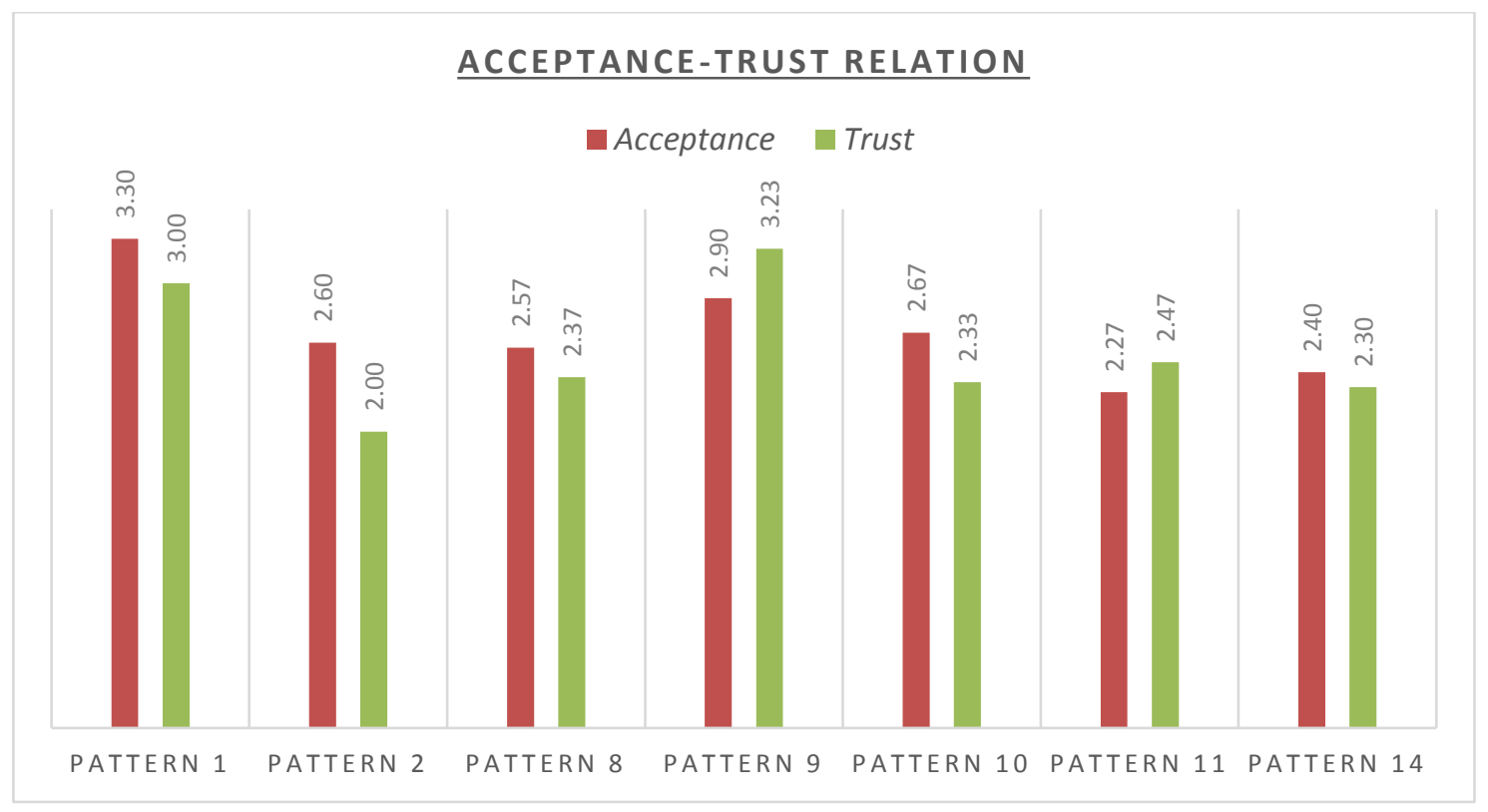

Figure 6: Noted Acceptance-Trust relationships in 7 patterns

Coates (2003) has proposed that highly ordered curved objects are more cognitively intelligible. By implication, the curved and symmetric properties of this structure may be a guiding factor in the positive emotive interpretation. Norman (2004) has characterised this as "visceral" design, where there is a naturally positive reaction to a composition based on a basic cognitive preference.

Another result we can consider within the spectrum of curved geometric elements is the illusion of movement. Gestalt theory suggests that the perception of shapes themselves is altered by the experience of the whole composition. The responses towards patterns 4, 6, 12 and 15 all contained high averaged scores for serenity $(>2.3)$ suggesting that they represented feelings of calmness to some extent. If we consider the compositions of patterns, all feature structured undulating lines with glide reflection symmetry (waves). Following the Gestalt theorists we can consider how this structural element may contextually lead to an illusion of movement — what Pinna (2010), mentioned earlier, described as a "happening" and Arnheim (1954) described as a psychological force. We suggest that this compositional structure relates to conceptual understanding of fluidity or running water. This aesthetic similarity to water may be 
leading, in part, to the high results for serenity. The flowing nature of water is a powerful cultural symbol (Clarke, 2010) and has long been associated with characteristically positive notions of peace, harmony or the "sublime" as described in the tradition of European Romanticism (Bietoletti, 2005).

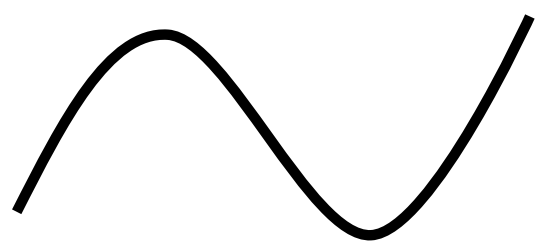

Figure 7: Undulating line motif can be interpreted as an abstract visual representation of flowing water

\section{Angularity in pattern is interesting and distracting}

Pattern that uses angularity as its principal aesthetic tool posed a different challenge of interpretation. While there was much coherence noted for the dominantly curved patterns, the results for angular patterns were more ambiguous. A particular relationship worth noting is between the experience of interest and that of distraction (Figure 8).

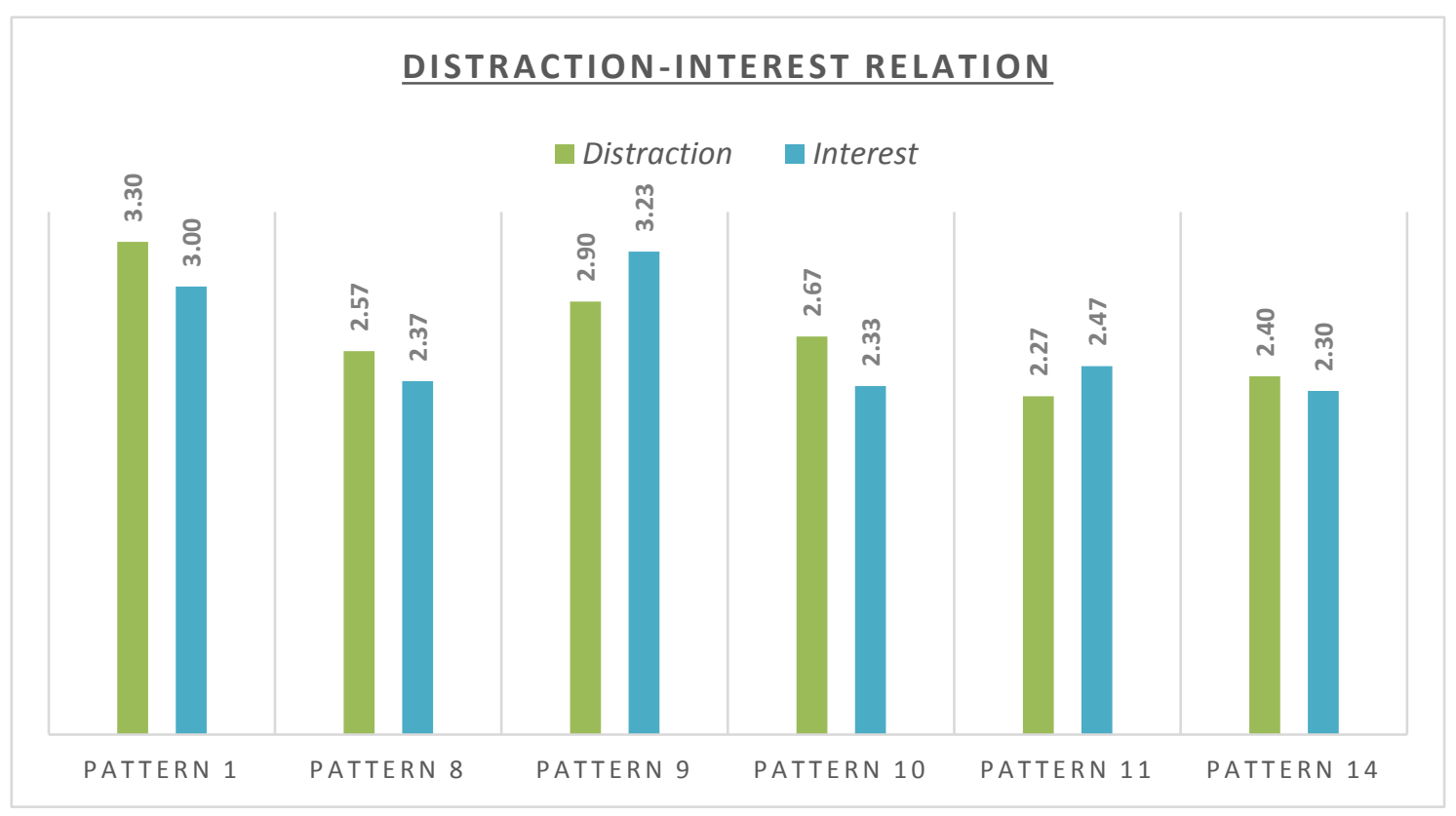

Figure 8: Noted Distraction-Interest relationships in 6 patterns 
This relationship was recorded in ten patterns, both within the curved and angular pattern categories. Our interest here concerns aspects of perceptual complexity. These results suggest that the context and composition of these angular forms make them more engaging or stimulating for observers. Pattern 1 and pattern 5 are both highly angular and both display this relationship between interest and distraction - interest being semantically more positive than distraction (within Plutchik's, 1980 framing). We propose that that the negative interpretation of the angularity is being curtailed by the compositional features of the pattern. Pattern 1 for instance appears visually comparable to a spiral and pattern 5 comparable to a maze or labyrinth (see Figure 9).

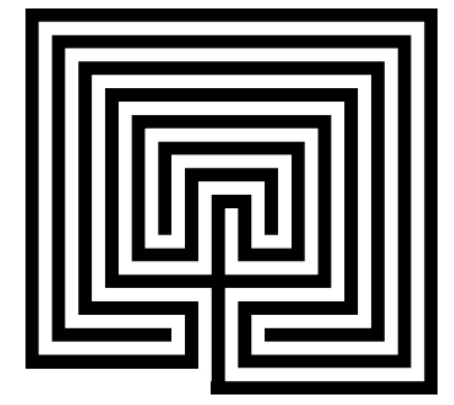

Figure 9: Plan of a Cretan labyrinth design is similar in appearance to some of the dominantly angular patterns (open-source image from Wikimedia Commons)

These cultural indicators may inspire more positive feelings of adventure through heightened visual stimulation. Similar maze and spiral motifs are seen elsewhere in the other angular patterns that show this same result, notably patterns 8 and 10 . As discussed earlier, Ingold (2008) and Gell (1998) have noted the historic use of pattern to warn off demonic forces. In a similar sense, contemporary observers may experience a subjective sense of confusion or mystery, though the absolute intensity of these experiences is not clear. A psychoanalytic assessment might say that people on some level are attracted to the unknown, that the angularity might present what Freud (1920) characterised as a desire for danger. This can be challenged however by noting the 
relatively high ratings for annoyance seen in the results for patterns $1,5,8$ and 10 - the visual composition may also be irritating. In some of these examples also, symmetry is less obviously detectable, meaning that the visual processing and intelligibility of the angular patterns may not be as smooth. Patterns 8,9 and 10 all use sophisticated symmetry structures that may not be immediately obvious to observers. Pattern 7 for example recorded the highest average for annoyance. Considering that this pattern is one of the most aesthetically complex, containing many overlapping lines with diverse directional change and a complex symmetry arrangement, it may present a challenge in cognitive processing and result in irritation (following Coates, 2003).

The ambiguous results of many of the patterns may be because of cultural symbolism offsetting naturally developed cognitive preferences. All the participants were of Western European and North American cultures meaning that the interpretation of the patterns is viewed through a distinct cultural lens. It is telling for example that Christian symbolism is interpreted as conveying trust - this may not be the case out with the bounds of a Western culture where the dominant religion is Christianity. Other examples, such as the positive interpretation of pattern number 13, may be due to its use of organic symbolism. In the Classical traditions of Western art, organic forms were used extensively as a means of ornamentation, and often as explicit symbols or icons [author(s)]. This may account for some of the positive interpretation observed here - as organic forms were so dominant and directly associated with a notion of "the sublime" and the beautiful. Similar organic motifs are indeed used in dominant aesthetic movements from the past, notably the Arts and Crafts and Art Nouveau movements whose practitioners developed hugely elaborate pattern designs utilising imagery from the natural world - the preeminent examples of this being William Morris and Antoni 
Gaudi (Pevsner, 1960). Pattern designs, in this sense, could relay a form of cultural nostalgia, what Norman (2004) has referred to as "reflective" design.

\section{Implications for design practice}

Considering that there is an established interest in designing for emotive experiences (Desmet, 2012; Desmet \& Hekkert, 2014), this work can inform the creation of more emotively sensitive products through the utilisation of psychologically and culturally attuned symbolism. As was previously stated, pattern is an emergent property of design and construction where a process of making will create a patterning effect. A woven basket, a knitted jumper, brick and stone work, mosaic and cartography all interface with patterning principles. Practices such as Kansai Engineering are directly orientated around creating emotively engaging and experientially attuned products through understanding cognition and psychology (Lévy, 2013), and a greater understanding of pattern could be applied within Kansei frameworks, focusing on aesthetic symbolism. Aesthetics being one of the principal factors in design styling and the emotional experience of products generally (Norman, 2004).

In terms of constructing an emotive pattern our insights offer some obvious starting points. Utilising methods that identify specific motifs, we can understand which aesthetic elements are the most important for achieving an emotive affect. Once an isolated geometry has been identified as emotively significant, this can then be configured into a bespoke pattern design. Variations in orientation and scaling may enhance or change the perceptual characteristics (following Foster, 1984), but the practice of motif isolation could be a valuable tool for design practitioners to create distinct patterns with attuned aesthetic symbolism - patterns that could be applied onto any number of products and artefacts. By extension, highly symbolic pattern could be used as a basis for texturing designs, creating both attuned aesthetic and tactile 
experiences for product users. Work by Karana, Hekkert and Kandachar (2009) has pointed out that the textural qualities of different materials can be linked to distinct emotive experiences. Precisely designed pattern-based texturing could be used to achieve similar affects, expanding the scope of this initial study.

\section{Conclusion}

This study has sought to determine if distinct emotive experiences could be linked to a set of culturally significant patterns. Firstly, we explored the definition, history and makeup of pattern and how they are bounded by structural rules. Following this, we examined the existing research concerning aesthetic perception, notably the Gestalt theorists, and explored the existing paradigm in vision research where aesthetic curvature is generally associated with a more positive emotional valence. An exploratory study was formulated in which the more dominantly curved patterns tended to be associated with positive emotions. Patterns with interlocking ring motifs were associated with concepts of trust and acceptance and patterns with wave motifs were associated with serenity. We argue these results are driven by cultural factors such as symbolism and the cognitive detection of symmetry. With patterns containing angular forms, there was an inclination towards an interpretation of negative emotive valence, though the results were more ambiguous overall. We suggest that the compositional nature of the angular forms influenced interpretation - many of the angular patterns were associated with high levels of distraction and interest, implying there is a level of positive stimulation for observers. It is also suggested that the strong cultural symbols of mazes, labyrinths and spirals play a role in the interpretations - the sense of danger or mystery may be stimulating and exciting to observers. This initial exploratory study helps to lay a foundation for future research into pattern, the possible development of bespoke emotive patterns and the refinement of pattern use within design practice. 


\section{References}

Arnheim, R. 1954. Art and Visual Perception: a psychology of the creative eye. London: Faber and Faber.

Arnold, M. B. 1960. Emotion and Personality, Volume 1. New York: Columbia University Press.

Bar, M., and Neta, M. 2006. "Humans prefer curved visual objects.” Psychological Science, 17(8), 645-648. http://doi.org/10.1111/j.1467-9280.2006.01759.x

Bertamini, M., L. Palumbo, T. N. Gheorghes, and M. Galatsidas. 2016. "Do observers like curvature or do they dislike angularity?" British Journal of Psychology, 107(1), 154-178. http://doi.org/10.1111/bjop.12132

Bietoletti, S. (2005). Neoclassicism and Romanticism. New York: Sterling Publishing.

Broug, E. 2013. Islamic geometric design. London: Thames \& Hudson.

Cassirer, E. 1962. An Essay on Man. New Haven: Yale University Press.

Christie, A. 1969. Pattern Design: An Introduction to the Study of Formal Ornament. Mineola, NY: Dover Publications, Inc.

Clarke, D. J.2010. Water and art: a cross-cultural study of water as subject and medium in modern and contemporary artistic practice. London: Reaktion Books.

Coates, D. 2003. Watches Tell More Than Time: Product Design, Information, and the Quest for Elegance. New York: McGraw-Hill Education.

Collier, G. L. 1996. “Affective synesthesia: Extracting emotion space from simple perceptual stimuli." Motivation and Emotion, 20(1), 1-32. http://doi.org/10.1007/BF02251005

Cruell, W., Mateiciucová, I., and Nieuwenhuyse, O. 2017. Painted Pots, Painting People: Late Neolithic Ceramics in Ancient Mesopatamia. Barnsley: Oxbow Books.

Desmet, P. M. A. 2003. “A multilayered model of product emotions.” The Design Journal, 4-13. http://doi.org/10.2752/146069203789355480

Desmet, P. M. A. 2012. "Faces of product pleasure: 25 positive emotions in humanproduct interactions." International Journal of Design, 6(2), 1-29.

Desmet, P. M. A., and Hekkert, P. 2014. "Special Issue Editorial: Design \& Emotion." International Journal of Design, 2(3), 1-6.

Dewey, J. 1934. Art As Experience. New York: Perigee Books.

Ekman, P. 1980. "Facial signs of emotional experience." Journal of Personality and Social Psychology, 39(6), 1125. 
Foster, D. H. 1984. 'Local and global computational factors in visual pattern recognition' in Figural Synthesis. eds. Dodwell, P.C. and Caelli, T. 83-115. Hillsdale: Lawrence Erlbaum Associates.

Frey, O., \& Schwappach, F. 1973. "Studies in early Celtic design." World Archaeology, 4:3, 339-356, doi: 10.1080/00438243.1973.9979544

Freud, S. 1920. Beyond the Pleasure Principle. London: Penguin Books.

Gell, A. 1998. Art and Agency: An Anthropological Theory. Oxford: Oxford University Press

Gómez-Puerto, G., E. Munar, and M. Nadal. 2015. "Preference for Curvature: A Historical and Conceptual Framework.” Frontiers in Human Neuroscience, 9, 712. http://doi.org/10.3389/fnhum.2015.00712Hann, M. 2012. Structure and Form in Design: Critical Ideas for Creative Practice. London: Bloomsbury Publishing.

Hann, M. A. 2013. Symbol, Pattern \& Symmetry: the cultural significance of structure. London: Bloomsbury Publishing.

Hekkert, P. 2006. "Design aesthetics: principles of pleasure in design Design aesthetics: principles of pleasure in design.” Psychology Science, 48(2), 157-172.

Hekkert, P., and Berghman, M. 2016. "The beauty of balance - An empirical integration of the Unified Model of Aesthetics for product design.” in 2016 Design Research Society 50th Anniversary Conference.

Hiscock, N. 2007. The Symbol at Your Door: Number and Geometry in Religious Architecture of the Greek and Latin Middle Ages. Abingdon: Routledge.

Hogarth, W. [1753] 1997. The Analysis of Beauty. New Haven: Yale University Press.

Hume, D. [1738] 1985. A Treatise of Human Nature. London: Penguin Books.

Ingold, T. 2008. Lines: a breif history. Abingdon, UK: Routledge.Ingold, T. 2009. "The textility of making." Cambridge Journal of Economics, 34(1), 91-102. http://doi.org/10.1093/cje/bep042

Ingold, T. 2015. The Life of Lines. Abingdon: Routledge.

Izard, C. E. 2009. "Emotion theory and research: Highlights, unanswered questions, and emerging issues." Annual Review of Psychology, 60(1), 1-25. http://doi.org/10.1146/annurev.psych.60.110707.163539

James, W. 1884. "What is an emotion?” Mind, 9(34), 188-205.

Jones, O. [1856] 2008. The Grammar of Ornament. London: Bloomsbury Publishing. 
Jordan, P. W. 2002. Designing Pleasurable Products: An Introduction to the New Human Factors. Boca Raton: CRC Press.

Jung, C. G. 1991. The Archetypes and the Collective Unconscious (Collected Works of C.G. Jung). Abingdon: Routledge.

Karana, E., P. Hekkert, and P. Kandachar. 2009. "Meanings of materials through sensorial properties and manufacturing processes.” Materials and Design, 30, $2778-2784$

Krippendorff, K. 2005. The Semantic Turn: A New Foundation for Design. Boca Raton: CRC Press.

Krippendorff, K., and Butter, R. 1984. "Product Semantics: Exploring the Symbolic Qualities of Form.” Innovations, 3, 4-9.

Lazarus, R. S. (1991). Emotion and adaptation. Oxford: Oxford University Press.

Leder, H., B. Belke, A. Oeberst, and D. Augustin. 2004. "A model of aesthetics appreciation and aesthetic judgment”. British Journal of Psychology, 95, 489508.

Leder, H., and Nadal, M. 2014. "Ten years of a model of aesthetic appreciation and aesthetic judgments: The aesthetic episode-Developments and challenges in empirical aesthetics". British Journal of Psychology, 105(4), 443-446. doi: 10.1111/bjop. 12084

Lévy, P. 2013. "Beyond Kansei Engineering: The emancipation of Kansei design." International Journal of Design, 7(2), 83-94.

Mitrovic, B. 1998. "Paduan Aristotelianism and Daniele Barbaro's Commentary on Vitruvius' De Architectura.” The Sixteenth Century Journal, 29(3), 667-688. doi: $10.2307 / 2543683$

Nanda, U., D. Pati, H. Ghamari, and R. Bajema. 2013. "Lessons from neuroscience: form follows function, emotions follow form." Intelligent Buildings International, 5(SUPPL1), 61-78.http://doi.org/10.1080/17508975.2013.807767

Norman, D. A. 2004. Emotional Design: Why We Love (or Hate) Everyday Things. New York, NY: Basic Books.

Palumbo, L., and Bertamini, M. 2016. "The Curvature Effect." Empirical Studies of the Arts, 34(1), 35-52. http://doi.org/10.1177/0276237415621185

Panofsky, E. 1939. Studies in Iconology. Oxford: Oxford University Press.

Pevsner, N. 1960. Pioneers of Modern Design: From William Morris to Walter Gropius. New Haven: Yale University Press. 
Pile, J. 2000. A History of Interior Design. London: Laurence King Publishing.

Pinna, B. 2010. "New Gestalt principles of perceptual organization: an extension from grouping to shape and meaning." Gestalt Theory, 23(1), 11-78.

Plutchik, R. 1980. Emotion, a psychoevolutionary synthesis. New York: Harper \& Row.

Poffenberger, A. T., and Barrows, B. E. 1924. "The Feeling Value of Lines.” Journal of Applied Psychology, 8(2), 187-205. http://doi.org/10.1037/h0073513

Russell, J. A. 2003. "Core Affect and the Psychological Construction of Emotion." Psychological Review, 110(1), 145-172. http://doi.org/10.1037/0033295X.110.1.145

Salgado-Montejo, A., C. J. Salgado, J. Alvarado, and C. Spence. 2017. “Simple lines and shapes are associated with, and communicate, distinct emotions." Cognition and Emotion, 31(3), 511-525. http://doi.org/10.1080/02699931.2015.1133401

Saussure, F. (2013). Course in General Linguistics. London: Bloomsbury Publishing. (original work published in 1916)

Scherer, K. R., A. Schorr, and T. Johnstone. 2001. Appraisal Processes in Emotion: Theory, Methods, Research. Oxford: Oxford University Press.

Shaver, P., J Schwartz, D. Kirson, and C. O’Connor. 1987. “Emotion knowledge: Further exploration of a prototype approach.” Journal of Personality and Social Psychology, 52(6), 1061-1086. http://doi.org/10.1037/0022-3514.52.6.1061

Silvia, P. J., and Barona, C. M. 2009. "Do People Prefer Curved Objects? Angularity, Expertise, and Aesthetic Preference." Empirical Studies of the Arts, 27(1), 2542. http://doi.org/10.2190/EM.27.1.b

[author(s)]

Wade, D. 1982. Geometric Patterns and Borders. London: Wildwood House Ltd.

Washburn, D. K., and Crowe, D. S. 1988. Symmetries of Culture: Theory and Practice of Plane Pattern Analysis. Washington, DC: University of Washington Press 